\title{
EPIDEMIOLOGÍA MOLECULAR DE AISLAMIENTOS DE ACINETOBACTER BAUMANNII EN LA CIUDAD DE GUAYAQUIL
}

ISOLATIONS OF ACINETOBACTER BAUMANNII USING MOLECULAR EPIDEMIOLOGY IN THE CITY OF GUAYAQUIL EPIDEMIOLOGIA MOLECULAR DO ISOLAMENTO DE ACINETOBACTER BAUMANNII NA CIDADE DE GUAYAQUIL

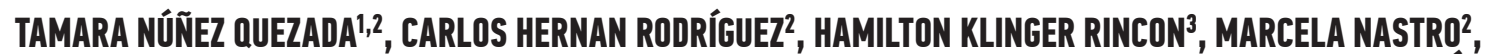 \\ NORA BALDERRAMA YARHUI', LAURA DABOS ${ }^{2}$, YESSENIA ACOSTA MOSQUERA', NATASHA PLAZA MOREIRA', ÁNGELA \\ FAMIGLIETTI ${ }^{2}$ \\ $\begin{array}{ll}1 & \text { Hospital Teodoro Maldonado Carbo, Guayaquil, Ecuador } \\ 2 & \text { Hospital José de San Martín, Buenos Aires, Argentina } \\ { }^{3} \text { Hospital de Infetología, José Daniel Rodríguez Maridueña, Guayaquil, Ecuador. }\end{array}$
}

RESUMEN

Objetivo: describir brote causado por acinetobacter baumannii recuperado en dos centros asistenciales de la ciudad de Guayaquil, mediante técnicas de epidemiología molecular. Materiales y métodos: treinta y tres aislamientos de A. baumannii fueron recuperados de dos centros médicos de la ciudad de Guayaquil, Ecuador, entre noviembre de 2012 y octubre de 2013. Los aislamientos fueron identificados mediante MALDI-TOF y por la presencia de blaOXA-51. El análisis epidemiológico se realizó mediante PCR. Resultados: 33 aislamientos fueron sensibles solo a colistina. En 29 se detectó OXA-24/40. La secuenciación del ADN identificó a blaOXA-24/40 como blaOXA-72. Todos presentaron el mismo patrón de PCR. Conclusión: se presenta el primer brote de blaOXA-72 en aislados de A. baumannii en América del sur. Este es el primer estudio llevado a cabo en la República de Ecuador.

PALABRAS CLAVE: acinetobacter baumannii, oxacilinasa, América, carbapenem-resistancia.

ABSTRACT

Objective: describe an outbreak caused by Acinetobacter baumannii recovered in two healthcare centers in the city of Guayaquil using molecular epidemiology techniques. Materials and methods: thirty-three isolates of A. baumannii recovered from two medical centers in the city of Guayaquil, Ecuador, between November 2012 and October 2013. The isolates were identified by MALDI-TOF and by the presence of blaOXA- 51. The epidemiological analysis was investigated by PCR. Results: 33 isolates were sensitive only to colistin. In 29, OXA-24/40 was detected. DNA sequencing identified blaOXA-24/40 as blaOXA-72. They all presented the same PCR pattern. Conclusions: we report the first blaOXA-72 outbreak in A. baumannii isolates in South America. This is the first study carried out in the Republic of Ecuador

KEYWORDS: acinetobacter baumannii, oxacillinase, America, carbapenem-resistance.

RESUMO

Objetivo: descrever um surto causado por acinetobacter baumannii recuperado em dois centros de saúde na cidade de Guayaquil utilizando técnicas de epidemiologia molecular. Materiais e métodos: trinta e três isolados de A. baumannii foram recuperados de dois centros médicos na cidade de Guayaquil, Equador, entre novembro de 2012 e outubro de 2013. Os isolados foram identificados por MALDI-TOF e pela presença de blaOXA- 51 A análise epidemiológica foi investigada por PCR Resultados: 33 isolados foram sensíveis apenas à colistina. Em 29 OXA-24/40 foi detectado. A sequenciação de DNA identificou blaOXA-24/40 como blaOXA-72. Todos eles apresentaram o mesmo padrão de PCR. Conclusões: relatamos o primeiro surto de blaOXA-72 em isolados de A. baumannii na América do Sul. Este é o primeiro estudo realizado na República do Equador.

PALAVRAS-CHAVE: acinetobacter baumannii, oxcilinase, América, resistência ao carbapenêmico. 
INTRODUCCIÓN

Probablemente el primer aislamiento de acinetobacter spp. comunicado en la literatura data de 1908 bajo el nombre de diplococcus mucosus. ${ }^{1} \mathrm{La}$ bacteria fue identificada por la ausencia de propiedades: incoloro, inmóvil, incapaz de reducir nitratos, oxidasa negativo y no fermentador de azucares. La falta de características distintivas se usó como ayuda en la nomenclatura: micrococcus (pequeño), achromobacter (sin color), acinetobacter (sin movimiento), anitratus (no reductor de nitratos). Actualmente el género acinetobacter spp. está constituido por 33 genoespecies. ${ }^{2}$ La imposibilidad de distinguirlas mediante pruebas bioquímicas ha dificultado el estudio de la epidemiología del género. ${ }^{3}$

Si bien, acinetobacter baumannii es la genoespecie aislada con mayor frecuencia, asociada en forma predominante a infecciones hospitalarias, recientemente se han comunicado varios trabajos en diferentes países en los cuales acinetobacter nosocomialis y acinetobacter pitti están presentes en altos porcentajes. ${ }^{4,5} \mathrm{~L}$ a resistencia a carbapenemes esta principalmente asociada a la producción de capapenemasas de clase $\mathrm{D}$ y dentro de ellas, OXA-23.6-8

En las últimas décadas las infecciones nosocomiales producidas por A. baumannii aumentaron de manera considerable en todo el mundo. Sin embargo, grandes diferencias se observan entre diferentes países. La multirresistencia, la capacidad de sobrevivir en superficies inanimadas son las principales característica de A. baumannii. ${ }^{3} \mathrm{Si}$ bien estas propiedades son comunes a todas las regiones, están influenciadas por características hospitalarias, culturales y por el uso de diferentes antibióticos, por lo que es indispensable vigilar la evolución de la resistencia a los antimicrobianos en cada país, establecer una casuística propia y evaluar las medidas adecuadas para disminuir su diseminación.

El objetivo de este trabajo fue describir un brote causado por A. baumannii resistentes a los carbapenemes recuperados en dos centros asistenciales de la ciudad de Guayaquil mediante técnicas de epidemiología molecular.

\section{METODOLOGÍA}

Se estudiaron en forma prospectiva los aislamientos de acinetobacter spp. resistentes a los carbapenemes recuperados de pacientes atendidos (internados y consultorio externo) en dos centros médicos (hospital IESS "Teodoro Maldonando Carbo"(R1); hospital de Infectología "José Daniel Rodríguez Maridueña" (R2)), de la ciudad de Guayaquil en el periodo comprendido entre noviembre de 2012 y octubre de 2013. Los aislamientos fueron identificados por características culturales y mediante la espectrometría de masa con un equipo MALDI-TOF, Bruker, Daltonics.

Los resultados fueron analizados utilizando la base de datos de Biotyper (versión 3.1, BD, Bruker Daltonik, Bremen, Alemania). La sensibilidad a los antimicrobianos fue realizada mediante el equipo VITEK-2(bioMe'rieux, Marcy L 'E'toile, Francia).

Se utilizaron las recomendaciones del Clinical and Laboratory Standards Institute (CLSI) en la interpretación de los resultados. ${ }^{9}$ La presencia de blaOXA-51, blaOXA-23, blaOXA-58 y blaOXA-24/40, se investigó mediante cebadores específicos y posterior secuenciación, de acuerdo a lo publicado previamente por Woodford. ${ }^{10} \mathrm{La}$ relación clonal entre los aislamientos se realizó mediante OD-PCR. ${ }^{11}$

Para cada paciente se elaboró una ficha clínica en donde se incluyeron los siguientes datos: espécimen clínico, tratamiento previo con carbapenemes, fecha y servicio de internación; enfermedad de base y / o diagnóstico. Se consideró una infección nosocomial cuando la misma se inicia en el paciente luego de 48 horas de internación.

\section{RESULTADOS}

En el periodo estudiado se recuperaron 33 aislamientos de acinetobacter spp resistentes a los carbapenemes (29 de los cuales pertenecen al centro R1). Todos fueron identificados como A. baumannii por espectrometría de masa y en la totalidad se detectó la presencia de blaOXA-51.

El 69\% de los pacientes fueron masculinos y todos los aislamientos fueron de origen intrahospitalario. El rango de edad de los pacientes fue 40-97 años con una media de 66 años. El tiempo promedio de internación previo a la recuperación por infección con A. baumannii fue de 19 días. Si bien, los pacientes fueron detectados en todos los servicios médicos, en el $68 \%$ se verificó la realización de algún tipo de cirugía. El 38,7\% de los mismos murieron dentro de los 30 días posteriores a la recuperación de A. baumannii, aunque no se determinó si la misma estaba directamente asociada a este hallazgo. 
Los aislamientos se recuperaron de los siguientes especímenes clínicos: 23 del tracto respiratorio inferior, 5 punción por piel sana, 4 de orina y 1 de punta de catéter (Tabla 1).

La sensibilidad a los antimicrobianos fue la siguiente: $100 \%$ a colistina, $58,6 \%$ a, ampicilinasulbactam, $7 \%$ a tetraciclina, mientras que la resistencia fue total a la ampicilina, ceftriaxona, ceftazidima, imipenem, meropenem, amikacina, gentamicina, trimetroprima-sulfametoxazol y ciprofloxacina, (Tabla 1).

De los 33 aislamientos de A. baumannii estudiados, se obtuvieron 3 tipos clonales: el predominante fue el clon A con 29 aislamientos. El gen blaOXA-24/40 se detectó en los 29 aislamientos pertenecientes al clon $\mathrm{A}$, mientras que, blaOXA-23 solo se detectó en 4 aislamientos, los cuales se encontraron 3 en el clon B y 1 en el clon C. La secuenciación del ADN identificó al amplicon blaOXA-24/40 como blaOXA-72, (Tabla 1).

\section{TABLA 1.PRINCIPALES CARACTERÍSTICASCLÍNICAS, MICROBIOLÓGICAS Y EPIDEMIOLÓGICAS DEL BROTE DEA. BAUMANNII ESTUDIADO} CARACTERÍSTICA ESTUDIADA

\begin{tabular}{|c|c|}
\hline Sexo masculino & $69 \%$ \\
\hline Edad promedio & 66 \\
\hline Rango de edades & $40-97$ \\
\hline DDIPP* & 19 \\
\hline Pacientes con cirugía previa & $68 \%$ \\
\hline Mortalidad a los 30 días & $38,7 \%$ \\
\hline \multicolumn{2}{|l|}{ \% de Sensibilidad a: } \\
\hline Colistina & 100 \\
\hline Ampicilina-sulbactam & 58,6 \\
\hline Tetraciclina & $7 \%$ \\
\hline bla0XA-51 & $33 / 33$ \\
\hline blaOXA-23 & $4 / 33$ \\
\hline bla0XA-72 & $29 / 33$ \\
\hline Clon predominante & $A: 29 / 33$ \\
\hline
\end{tabular}

DISCUSIÓN

En los últimos años, las infecciones nosocomiales causadas por acinetobacter baumannii en diferentes centros asistenciales han pasado de causar brotes esporádicos a ser hiperendémico, sobre todo en las unidades de cuidados intensivos. ${ }^{3}$ Los factores de riesgo para la adquisición de $\mathrm{A}$. baumannii se identificaron generalmente a partir de brotes epidémicos. ${ }^{12}$ En situaciones endémicas como la que se verifica en nuestras instituciones, la epidemiologia cambia. En estas circunstancias la importancia de los reservorios ambientales es relativa y los pacientes ya colonizados constituyen los principales focos de diseminación. ${ }^{12}$ En forma coincidente a lo previamente descrito, se determina en este trabajo que la cirugía previa es uno de los principales factores de riesgo (68\%) para la infección/ colonización por A. baumannii. ${ }^{13}$

Diferentes estudios coinciden en que la gran mayoría de los pacientes se colonizan por A. baumannii en la primera semana de internación. ${ }^{12,13}$ En el presente trabajo el periodo previo promedio fue superior (19 días), pero en la mitad de los pacientes el tiempo fue inferior a los 10 días, evidenciando un alto grado de "presión de colonización" (prevalencia de pacientes colonizados). Cabe mencionar que en todos los pacientes incluidos en el estudio, la adquisición de $\mathrm{A}$. baumannii resistente a los carbapenemes fue de origen intrahospitalario.

La fuerte asociación de A. baumannii con la infección respiratoria baja intrahospitalaria IH ya ha sido extensamente descrita. ${ }^{3,11,13}$ Estos aislamientos se recuperaron en forma continua en todo el periodo estudiado (dato no mostrado) evidenciando la endemia de las infecciones IH por A. baumannii.

En la epidemiología de A. baumannii la falta de resolución de los reservorios de los primeros brotes epidémicos trae aparejado una mayor dificultad en su erradicación y una mayor multiplicación de dichos microorganismos; en consecuencia la epidemia se transforma en endemia; emergen nuevos mecanismos de resistencia por la presión selectiva ejercida por el uso de antimicrobianos y se asientan clones de comportamiento epidémico que se dispersan no solo en las diferentes salas del hospital, sino también en una amplia zona geográfica. En esta situación la resistencia a carbapenemes es uno de los últimos peldaños en la multirresistencia de A. baumannii ya que frecuentemente se suma, como se observó en las cepas etectadas, la resistencia a otros antimicrobianos (cefalosporinas, aminoglucósidos, fluoroquinolonas).

En forma similar a otros trabajos, en el presente se determina a la colistina como el antimicrobiano con mayor actividad frente a A. baumannii-MR. OXA-23 es la carbapenemasa predominante y este fenómeno se acentuó en los últimos 5 años en el mundo y también en Sud América. ${ }^{8}$ Sin embargo, en el estudio se determinó la presencia mayoritaria de OXA-72. Esta carbapenemasa pertenece al subgrupo OXA -24/407. El primer informe de OXA72, ocurrió en Tailandia en 2004 y posteriormente 
fue identificado en Taiwán, China y Corea del Sur y en algunos países europeos. ${ }^{14-17}$ En América sólo aislamientos esporádicos con blaOXA-72 (tanto A. baumannii como de A. pitti) fueron reportados. ${ }^{18,19}$

Los aislamientos carbapenem sensibles (por lo tanto no forman parte de los objetivos de este trabajo) y los aislamientos con blaOXA-72 son indistinguibles con la técnica OD-PCR; mientras que los 4 productores de OXA-23, corresponden a 4 tipos clonales diferentes. Este patrón evidenciaría una diseminación de blaOXA-72 entre la población de A. baumannii carbapenem sensible y que este fenómeno se observe en los dos centros asistenciales de Guayaquil que intervinieron en el estudio, revela la diseminación de esta carbapenemasa, por lo menos en esta ciudad. Por lo tanto, sedetectó el primer brote de esta carbapenemasa en el continente americano.

No se pudo, mediantela certificación de viajes a las ciudades involucradas, de pacientes y / o personal de la salud, determinar la llegada de blaOXA-72. Los casos esporádicos publicados en América del Sur se sitúan en Guayaquil a una distancia promedio de $700 \mathrm{~km} .{ }^{18,19}$ En una segunda etapa nos avocaremos a coordinar un estudio nacional que permita valorar realmente su presencia en Ecuador, ya que según la información que disponen los autores, el presente es el primer estudio de este tipo realizado en el país.

\section{CONCLUSIÓN}

La resistencia a carbapenem en A. baumanii-MR, limita las opciones terapéuticas y la persistencia de los clones por largos períodos, lo que refleja una excelente adaptación del microorganismo al medio hospitalario. La presencia de blaOXA-72 como responsable de la resistencia a carbapenem en el brote descrito, es el primero que se reporta en el continente americano.

\section{REFERENCIAS BIBLIOGRÁFICAS}

1. Cowan ST. Unusual infections following cerebral operations: With a description of Diplococcus mucosus (von Lingelsheim). Lancet 1938; 2: 1052-4.

2. Peleg AY, Seifert H, and Paterson D. Acinetobacterbaumannii: Emergence of successful pathogen. Clin Microbiol Rev. 2008; 21:538-592.

3. Perez F., Hujer A., Hujer K., Decker B., Rather P., Bonomo R. Global challenge of multidrug-resistant Acinetobacter baumannii Antimicrob Agents Chemother. 2007; 51:3471-84.

4. Karah N, Haldorsen B, Hegstad K, Simonsen GS, Sundsford A, Samuelsen O. Species identification and molecular characterization of Acinetobacter spp. blood culture isolates from Norway. J Antimicrob Chemother. 2011;66:738-44.

5. Chuang YC, Sheng WH, Li SY, Lin YC, Wang JT, Chen YC, Chang SC. Influence of genospecies of Acinetobacter baumannii complex on clinical outcomes of patients with Acinetobacter bacteremia. Clin Infect Dis. 2011; 52:352-60

6. Poirel L, Naas T, Nordmann P. Diversity, epidemiology, and genetics of class $\mathrm{D} \beta$-lactamases. Antimicrob Agents Chemother 2010 ; 54:24-38.

7. Evans B and Amyes S. OXA $\beta$-Lactamases. Clin. Microbiol. Rev. 2014, 27:241.

8. Mugnier P, Poirel L, Naas T, Nordmann P. Worlwide dissemination if the blaoxa23 carbapenemase gene of Acinetobacter baumannii Emerging infection diseases 2010; 16(1):35-40.

9. Clinical Laboratory Standards Institute 2012. Performance Standards for Antimicrobial Susceptibility Testing; 22nd Informational Supplement, M100-S22. Wayne, Pa: Clinical and Laboratory Standards Institute.

10. Woodford N, Ellington M, Coehlo J, Turton J, Ward M., Brown S Amyes SG, Livermore DM. Multiplex PCR for genes encoding prevalent OXA carbapenemases in Acinetobacter spp. Int JAntimicrob Agents 2006; 27: 351-3.

11. Quelle LS, Catalano M. Efficacy of two DNA fingerprinting methods for typing Acinetobacter baumannii isolates. Diagn Microbiol Infect Dis. 2001; 39: 215-23.

12. Alvarez-Lerma F., Palomar M., Insuasti J., Olaechea P., Cerda E., Castillo F., Martinez-Pellu A. Infecciones por Acinetobacter spp. en pacientes criticos en UCI. Enferm Infecc Microbiol Clin 2005; 23: 533-9.

13. Martinez-Pellus A., Ruiz Gomez J., Sánchez F., Cordoba E., Fernández Lozana J. Incidencia de la colonización e infección por Acinetobacter baumannii en una UCI con situación de endemia. Análisis de factores de riesgo mediante un estudio de vigilancia. Enferm Infecc Microbiol Clin 2002; 5: 194-9.

14. Playford E., Craig J., Iredell J. Carbapenem-resistant Acinetobacter baumannii in intensive care unit patients: risk factors for acquisition, infection and their consequences. J. Hosp Infect 2007; 65: 204-11.

15. Wang H, Guo P, Sun H et al. Molecular epidemiology of clinical isolates of carbapenem-resistant Acinetobacter spp. from Chinese hospitals. Antimicrob Agents Chemother 2007; 51:4022-8.

16. Lee K, Kim MN, Choi TY, et al. Wide dissemination of OXA-type carbapenemases in clinical Acinetobacter spp. isolates from South Korea. Int J Antimicrob Agents 2009; 33: 520-4.

17. Barnaud G, Zihoune N, Ricard JD et al. Two sequential outbreaks caused by multidrug-resistant Acinetobacter baumannii isolates producing OXA-58 or OXA-72 oxacillinase in an intensive care unit in France. J Hosp Infect 2010; 76:358-60. 